\title{
ON ONE LEONTIEV-LEVIN THEOREM
}

\section{A.S. KRIVOSHEYEV, A.F. KUZHAEV}

\begin{abstract}
In this work we study the relations between different densities of a positive sequence and related quantities. More precisely, in the work we consider the upper density, the maximal density introduced by G. Polya, the logarithmic block density, which seems to be introduced first by L.A. Rubel. In particular, there were obtained relations between the maximal density and a quantity being very close to the logarithmic block density. The results of these studies are applied for generalizing the classical statement obtained independently by A.F. Leont'ev B.Ya. Levin on the completeness in a convex domain of a system of exponential monomials with positive exponents; we generalized this statement for the exponents with no density. We find out that for the aforementioned result, one can weaken the condition of the measurability of the sequence (that is, the existence of a density) and replace it by the identity of upper and maximal densities. Namely, we obtain a condition under which there holds the criterion of the completeness of the system of exponential monomials in convex domains. It should be noted that this criterion holds in a rather wide class of convex domains, for instance, having vertical and horizontal symmetry axes. The main role in solving this issues was played by the results of the studies by L.A. Rubel and P. Malliavin on relation between the growth of an entire function of exponential type along the imaginary axis and the logarithmic block density of its positive zeroes. These results were applied by these authors for studying the completeness of the system of exponentials in a horizontal strip.
\end{abstract}

Keywords: density of sequence, entire function, completeness, convex domain.

Mathematics Subject Classification: 30D10

Let $\Lambda=\left\{\lambda_{n}, m_{n}\right\}_{n=1}^{\infty}$ be a multiple sequence of positive numbers. Here $\left\{\lambda_{n}\right\}_{n=1}^{\infty}$ is an unbounded strictly increasing sequence and $m_{n}$ is the natural number called the multiplicity of an element $\lambda_{n}, n \geqslant 1$. We recall that the upper density and lower density of the sequence $\Lambda$ are respectively the quantities

$$
\bar{n}(\Lambda)=\varlimsup_{t \rightarrow+\infty} \frac{n(t, \Lambda)}{t}, \quad \underline{n}(\Lambda)=\varliminf_{t \rightarrow+\infty} \frac{n(t, \Lambda)}{t},
$$

where

$$
n(t, \Lambda)=\sum_{\lambda_{n} \leqslant t} m_{n}
$$

is the counting function of the sequence $\Lambda$, that is, the number of its elements counting the multiplicities located in the semi-interval $(0, t]$. If $\bar{n}(\Lambda)=\underline{n}(\Lambda)$, the sequence $\Lambda$ is called measurable and the quantity

$$
n(\Lambda)=\lim _{t \rightarrow+\infty} \frac{n(t, \Lambda)}{t}
$$

is well-defined and called the density of the sequence $\Lambda$.

We let $\mathcal{E}(\Lambda)=\left\{z^{k} e^{\lambda_{n} z}\right\}_{n=1, k=0}^{\infty, m_{n}-1}$. We shall say that a system of functions $\mathcal{E}(\Lambda)$ is complete in a convex domain $D \subseteq \mathbb{C}$ if it is complete in the space $H(D)$ of functions analytic in the domain $D$ with the topology of uniform convergence on compact subsets in $D$.

A.S. Krivosheyev, A.F. Kuzhaev, On one Leontiev-Levin theorem.

(c) Krivosheyev A.S., Kuzhaev A.F. 2017.

Submitted March 30, 201\%. 
The issue on completeness of the system $\mathcal{E}(\Lambda)$ in a convex domain $D$ was studied in many works (see, for instance, [1-3] and others). A.F. Leont'ev [4] and B.Ya. Levin [5, Ch. IV, Thm. 21] obtained independently the following classical result, which we formulate for the case of convex domains.

Let $D$ be a convex domain. A vertical diameter of domain $D$ is

$$
d(D)=\sup _{x} \sup _{\left(y_{1}, y_{2}\right)}\left\{\left|y_{1}-y_{2}\right|: z_{1}=x+i y_{1}, z_{2}=x+i y_{2}, \quad z_{1}, z_{2} \in D, \quad x, y_{1}, y_{2} \in \mathbb{R}\right\} .
$$

If $\Lambda$ is a measurable sequence with a density $n(\Lambda)=\tau>0$, then the system $\mathcal{E}(\Lambda)$ is complete in each convex domain with a vertical diameter satisfying $d(D) \leqslant 2 \pi \tau$ and is incomplete in each convex domain with a vertical diameter obeying $d(D)>2 \pi \tau$.

The aim of the present work is to generalize this result for the sequences without density in a wide class of convex domain, in particular, in the classes of domain having vertical and horizontal symmetry axes.

The mentioned generalization is based on the studies on relation between various densities of a positive sequence. We recall the definition of these densities.

First of all we need a characteristics introduced by G. Pólya [6], the maximal density of the sequence $\Lambda$ :

$$
\bar{n}_{0}(\Lambda)=\lim _{\delta \rightarrow+0} \varlimsup_{t \rightarrow+\infty} \frac{n(t, \Lambda)-n(t(1-\delta), \Lambda)}{\delta t}, \quad \delta \in(0 ; 1) .
$$

According the lemma in Section E3, Chapter IV in book [7], the limit as $\delta \rightarrow+0$ always exists and the maximal density is well-defined.

The logarithmic block density (or simply logarithmic density) $\bar{L}(\Lambda)$ of a positive sequence $\Lambda$ is

$$
\bar{L}(\Lambda)=\inf _{a>1} \varlimsup_{t \rightarrow+\infty} \frac{\lambda(a t)-\lambda(t)}{\ln a}, \quad \lambda(t)=\sum_{\lambda_{n} \leqslant t} \frac{m_{n}}{\lambda_{n}} .
$$

According to Lemma 3.2 of work [1], the quantity $\bar{L}(\Lambda)$ can be calculated as follows:

$$
\bar{L}(\Lambda)=\lim _{a \rightarrow+\infty} \varlimsup_{t \rightarrow+\infty} \frac{\lambda(a t)-\lambda(t)}{\ln a}
$$

that is, the limit as $a \rightarrow+\infty$ exists. In what follows we shall employ exactly this identity while working with the logarithmic density. Making a change of variables in the latter identity, we can write

$$
\bar{L}(\Lambda)=\lim _{\delta \rightarrow 1-0} \varlimsup_{t \rightarrow+\infty} \frac{\lambda(t)-\lambda(t(1-\delta))}{-\ln (1-\delta)},
$$

where $\delta \in(0 ; 1)$. We shall also make use of the following quantities:

$$
\begin{array}{ll}
\bar{L}(\Lambda, \delta)=\varlimsup_{t \rightarrow+\infty} \frac{\lambda(t)-\lambda(t(1-\delta))}{-\ln (1-\delta)}, & \delta \in(0 ; 1), \\
\bar{n}_{0}(\Lambda, \delta)=\varlimsup_{t \rightarrow+\infty} \frac{n(t)-n(t(1-\delta))}{\delta t}, \quad \delta \in(0 ; 1) .
\end{array}
$$

Employing these notations, we obtain

$$
\bar{L}(\Lambda)=\lim _{\delta \rightarrow 1-0} \bar{L}(\Lambda, \delta), \quad \bar{n}_{0}(\Lambda)=\lim _{\delta \rightarrow+0} \bar{n}_{0}(\Lambda, \delta) .
$$

The following statement holds.

Lemma 1. Assume that a sequence $\Lambda$ has a finite maximal density. Then the chain of the inequalities

$$
\underline{n}(\Lambda) \leqslant \bar{L}(\Lambda) \leqslant \bar{n}(\Lambda) \leqslant \bar{n}_{0}(\Lambda, \delta) \leqslant \bar{n}_{0}(\Lambda), \quad \delta \in(0 ; 1) .
$$

holds. 
Proof. The inequalities $\bar{n}(\Lambda) \leqslant \bar{n}_{0}(\Lambda, \delta) \leqslant \bar{n}_{0}(\Lambda), \delta \in(0 ; 1)$ were proved in [8, Lm. 2.1]. Let us prove the inequalities $\underline{n}(\Lambda) \leqslant \bar{L}(\Lambda) \leqslant \bar{n}(\Lambda)$.

Since $\bar{n}_{0}(\Lambda)<+\infty$, then $\bar{n}(\Lambda)<+\infty$. Then for each $\varepsilon>0$ there exists $r_{\varepsilon}$ such that for each $r \geqslant r_{\varepsilon}$ the inequality

$$
n(r, \Lambda)<(\bar{n}(\Lambda)+\varepsilon) r
$$

holds true. Let $\delta \in(0 ; 1)$ and $t(1-\delta) \geqslant r_{\varepsilon}$. Then by the previous inequality we obtain

$$
\begin{aligned}
\sum_{t(1-\delta)<\lambda_{n} \leqslant t} \frac{m_{n}}{\lambda_{n}} & =\int_{t(1-\delta)}^{t} \frac{d n(r, \Lambda)}{r}=\frac{n(t, \Lambda)}{t}-\frac{n(t(1-\delta), \Lambda)}{t(1-\delta)}+\int_{t(1-\delta)}^{t} \frac{n(r, \Lambda) d r}{r^{2}} \\
& <\frac{n(t, \Lambda)}{t}-\frac{n(t(1-\delta), \Lambda)}{t(1-\delta)}+(\bar{n}(\Lambda)+\varepsilon) \int_{t(1-\delta)}^{t} \frac{d r}{r} \\
& \leqslant \frac{n(t, \Lambda)}{t}+(\bar{n}(\Lambda)+\varepsilon) \int_{t(1-\delta)}^{t} \frac{d r}{r}<\bar{n}(\Lambda)+\varepsilon+(\bar{n}(\Lambda)+\varepsilon) \ln \left(\frac{1}{1-\delta}\right) \\
& =(\bar{n}(\Lambda)+\varepsilon)(1-\ln (1-\delta)) .
\end{aligned}
$$

Thus, in view of the arbitrariness of the number $\varepsilon>0$, by (3) we have

$$
\bar{L}(\Lambda, \delta) \leqslant \bar{n}(\Lambda) \frac{1-\ln (1-\delta)}{-\ln (1-\delta)}
$$

By (2) this implies the inequality $\bar{L}(\Lambda) \leqslant \bar{n}(\Lambda)$.

Employing the definition of the lower density, as in (6) we obtain

$$
\begin{aligned}
\sum_{t(1-\delta)<\lambda_{n} \leqslant t} \frac{m_{n}}{\lambda_{n}}>-\frac{n(t(1-\delta), \Lambda)}{t(1-\delta)}+(\underline{n}(\Lambda)-\varepsilon) \int_{t(1-\delta)}^{t} \frac{d r}{r} \\
>-\bar{n}(\Lambda)-\varepsilon+(\underline{n}(\Lambda)-\varepsilon) \ln \left(\frac{1}{1-\delta}\right), \quad \delta \in(0 ; 1), \quad t(1-\delta) \geqslant r_{\varepsilon} .
\end{aligned}
$$

By the arbitrariness of the number $\varepsilon>0$ and identity this follows that $\underline{n}(\Lambda) \leqslant \bar{L}(\Lambda)$. The proof is complete.

Remarks. 1. According (5) and [8, Lm. 2.1], if the sequence $\Lambda$ is measurable, the identities

$$
\underline{n}(\Lambda)=\bar{L}(\Lambda)=\bar{n}(\Lambda)=\bar{n}_{0}(\Lambda, \delta)=\bar{n}_{0}(\Lambda), \quad \delta \in(0 ; 1),
$$

hold true.

2. According (1) and (3), the identity

$$
\bar{L}(\Lambda) \leqslant \bar{L}(\Lambda, \delta), \quad \delta \in(0 ; 1) .
$$

holds.

In view of this and chain of inequalities (5), a natural question arises: whether the identity $\bar{L}(\Lambda, \delta) \leqslant \bar{n}(\Lambda), \delta \in(0 ; 1)$, is true. The positive answer would extend chain (5). However, the mentioned inequality is wrong. Let us consider the corresponding example.

Let $\delta \in(0 ; 1), 0<R_{k} \rightarrow \infty, k \rightarrow \infty$, and $R_{k+1} / R_{k} \rightarrow \infty, k \rightarrow \infty$. We define $\Lambda=\bigcup_{k \in \mathbb{N}} \Lambda_{k}$, where $\Lambda_{k}$ is the set consisting of all natural numbers in the interval $\left((1-\delta) R_{k}, R_{k}\right), k \in \mathbb{N}$; the multiplicity of each such number is equal to one. By the choice of the numbers $R_{k}$ we have

$$
\bar{n}(\Lambda)=\varlimsup_{t \rightarrow+\infty} \frac{n(t, \Lambda)}{t}=\lim _{k \rightarrow \infty} \frac{n\left(R_{k}, \Lambda\right)}{R_{k}} \leqslant \lim _{k \rightarrow \infty} \frac{R_{k-1}+\delta R_{k}}{R_{k}}=\delta .
$$


On the other hand,

$$
\bar{n}(\Lambda)=\lim _{k \rightarrow \infty} \frac{n\left(R_{k}, \Lambda\right)}{R_{k}} \geqslant \lim _{k \rightarrow \infty} \frac{\delta R_{k}}{R_{k}}=\delta .
$$

Thus, $\bar{n}(\Lambda)=\delta$. Moreover,

$$
\underline{n}(\Lambda)=\lim _{k \rightarrow \infty} \frac{n\left(R_{k}(1-\delta), \Lambda\right)}{R_{k}(1-\delta)} \leqslant \lim _{k \rightarrow \infty} \frac{R_{k-1}}{R_{k}(1-\delta)}=0 .
$$

Therefore, $\underline{n}(\Lambda)=0$. Let $\alpha \in(0 ; \delta]$. Then

$$
\begin{aligned}
& \bar{n}_{0}(\Lambda, \alpha)=\lim _{k \rightarrow \infty} \frac{n\left(R_{k}, \Lambda\right)-n\left(R_{k}(1-\alpha), \Lambda\right)}{\alpha R_{k}}=\lim _{k \rightarrow \infty} \frac{\alpha R_{k}}{\alpha R_{k}}=1, \\
& \bar{n}_{0}(\Lambda)=\lim _{\alpha \rightarrow 0} \bar{n}_{0}(\Lambda, \alpha)=1 .
\end{aligned}
$$

Moreover,

$$
\bar{L}(\Lambda, \alpha)=\lim _{k \rightarrow \infty} \frac{\lambda\left(R_{k}\right)-\lambda\left(R_{k}(1-\alpha)\right)}{-\ln (1-\alpha)} .
$$

By the Euler formula we have

$$
\lambda\left(R_{k}\right)-\lambda\left(R_{k}(1-\alpha)\right)=\sum_{R_{k}(1-\alpha)<n \leqslant R_{k}} \frac{1}{n}=\ln \frac{\left[R_{k}\right]}{\left[R_{k}(1-\alpha)+1\right]}+r_{k},
$$

where $[a]_{-}$is the integer part of a number a and $r_{k} \rightarrow 0, k \rightarrow \infty$. By the latter identity and (9) we get: $\bar{L}(\Lambda, \alpha)=1, \alpha \in(0 ; \delta]$. Assume now that $\alpha \in(\delta ; 1)$. Then identity (9) holds but at that,

$$
\lambda\left(R_{k}\right)-\lambda\left(R_{k}(1-\alpha)\right)=\sum_{R_{k}(1-\delta)<n \leqslant R_{k}} \frac{1}{n}=\ln \frac{\left[R_{k}\right]}{\left[R_{k}(1-\delta)+1\right]}+\tilde{r}_{k},
$$

where $\tilde{r}_{k} \rightarrow 0, k \rightarrow \infty$. Therefore,

$$
\bar{L}(\Lambda)=\lim _{\alpha \rightarrow 1-0} \bar{L}(\Lambda, \alpha)=\lim _{\alpha \rightarrow 1-0} \frac{-\ln (1-\delta)}{-\ln (1-\alpha)}=0 .
$$

Thus, in the considered examples the relations

$$
\underline{n}(\Lambda)=0=\bar{L}(\Lambda)<\delta=\bar{n}(\Lambda)<1=\bar{L}(\Lambda, \alpha)=\bar{n}_{0}(\Lambda, \alpha)=\bar{n}_{0}(\Lambda), \quad \alpha \in(0 ; \delta]
$$

hold true.

3. Assume that the sequence $\Lambda$ is such that its upper and maximal densities are finite and coincide: $\bar{n}(\Lambda)=\bar{n}_{0}(\Lambda)=\tau<+\infty$. Then it follows from (5) that $\bar{n}_{0}(\Lambda, \delta)=\tau, \delta \in(0 ; 1)$.

It turns out that the statement opposite to that in the above Remark 3 is true. In order to do it, we prove an auxiliary statement.

Lemma 2. Let $\bar{n}(\Lambda)<+\infty$. The identities hold:

$$
\bar{n}(\Lambda)=\bar{n}(\Lambda, 1)=\lim _{\delta \rightarrow 1-0} \bar{n}_{0}(\Lambda, \delta) .
$$

Proof. The identity $\bar{n}(\Lambda)=\bar{n}(\Lambda, 1)$ is implied immediately by (4).

Let $\delta_{k} \in(0 ; 1), k \in \mathbb{N}$, be a sequence such that $\delta_{k} \rightarrow 1, k \rightarrow \infty$. We have

$$
\begin{aligned}
0 & \leqslant \bar{n}_{0}\left(\Lambda, \delta_{k}\right)-\bar{n}_{0}(\Lambda, 1)=\varlimsup_{t \rightarrow+\infty} \frac{n(t)-n\left(t\left(1-\delta_{k}\right)\right)}{\delta_{k} t}-\bar{n}_{0}(\Lambda, 1) \\
& \leqslant \varlimsup_{t \rightarrow+\infty} \frac{n(t)}{\delta_{k} t}-\bar{n}_{0}(\Lambda, 1)=\frac{1}{\delta_{k}} \bar{n}(\Lambda)-\bar{n}_{0}(\Lambda, 1)=\bar{n}(\Lambda) \frac{1-\delta_{k}}{\delta_{k}} \rightarrow 0, \quad k \rightarrow \infty .
\end{aligned}
$$

Therefore, $\bar{n}_{0}\left(\Lambda, \delta_{k}\right) \rightarrow \bar{n}(\Lambda)$ as $\delta_{k} \rightarrow 1, k \rightarrow \infty$. The proof is complete.

Lemma 2, Remark 3 to Lemma 1 and the definition of the maximal density imply 
Corollary 1. The following statements are equivalent:

1) $\bar{n}(\Lambda)=\bar{n}_{0}(\Lambda)=\tau<+\infty$;

2) $\bar{n}_{0}(\Lambda, \delta)=\tau<+\infty, \delta \in(0 ; 1)$.

In the example in Remark 2 to Lemma 1 the identity $\bar{L}(\Lambda, \alpha)=\bar{n}_{0}(\Lambda)$ was obtained provided $\alpha \in(0 ; \delta]$. This suggests another equivalent definition of the maximal density by means of $\bar{L}(\Lambda, \alpha)$. We first prove an auxiliary statement.

Lemma 3. Let $\delta \in(0 ; 1)$. The inequalities

$$
\frac{-\delta}{\ln (1-\delta)} \bar{n}_{0}(\Lambda, \delta) \leqslant \bar{L}(\Lambda, \delta) \leqslant \frac{\delta}{(\delta-1) \ln (1-\delta)} \bar{n}_{0}(\Lambda, \delta)
$$

hold true.

Proof. We have

$$
\frac{n(t, \Lambda)-n(t(1-\delta), \Lambda)}{\delta t} \leqslant \frac{1}{\delta} \sum_{t(1-\delta)<\lambda_{n} \leqslant t} \frac{m_{n}}{\lambda_{n}}
$$

This yields:

$$
\bar{n}_{0}(\Lambda, \delta) \leqslant \frac{-\ln (1-\delta)}{\delta} \bar{L}(\Lambda, \delta)
$$

This implies the first inequality in (10). Let us prove the second inequality. We have

$$
\frac{1}{-\ln (1-\delta)} \sum_{t(1-\delta)<\lambda_{n} \leqslant t} \frac{m_{n}}{\lambda_{n}} \leqslant \frac{1}{-\ln (1-\delta)} \frac{n(t, \Lambda)-n(t(1-\delta), \Lambda)}{(1-\delta) t} .
$$

Therefore,

$$
\bar{L}(\Lambda, \delta) \leqslant \frac{\delta}{(\delta-1) \ln (1-\delta)} \bar{n}_{0}(\Lambda, \delta)
$$

The proof is complete.

Theorem 1. The identity

$$
\bar{n}_{0}(\Lambda)=\lim _{\delta \rightarrow+0} \bar{L}(\Lambda, \delta)
$$

holds true.

The statement of the theorem is implied immediately by the definition of $\bar{n}_{0}(\Lambda)$ and inequalities (10).

Let us provide one more equivalent definition of the maximal density. In order to do it, we need to get an additional information about the relations between $\bar{L}(\Lambda, \delta)$ and $\bar{n}_{0}(\Lambda)$.

Let $\delta \in(0 ; 1)$ and $\left\{t_{k}\right\}_{k=1}^{\infty}$ be an increasing sequence such that $0<t_{k} \rightarrow \infty$. We choose a natural number $p$ by the conditions $\delta /((1-\delta) p)<1, p>1$. Following [8], we let

$$
\alpha_{l}:=1-\frac{(l-1) \delta}{p}, \quad t_{k, l}:=\alpha_{l} t_{k}, \quad \delta_{l}:=\frac{\delta}{\alpha_{l} p}, \quad l=\overline{1, p} .
$$

By the choice of the number $p$ we have $\delta_{l} \in(0 ; 1), \delta_{l}<1, l=\overline{1, p}$. For each $k \in \mathbb{N}$, the semiinterval $\left(t_{k}(1-\delta) ; t_{k}\right]$ is partitioned into $p$ semi-intervals of form $\left(t_{k, l}\left(1-\delta_{l}\right) ; t_{k, l}\right]$. The length of each of them is in $p$ times less than the length of the original semi-interval $\left(t_{k}(1-\delta) ; t_{k}\right]$.

Lemma 4. The inequality

$$
\bar{L}(\Lambda, \delta) \leqslant \bar{n}_{0}(\Lambda), \quad \delta \in(0 ; 1)
$$

holds true. 
Proof. It is clear that we can assume that $\bar{n}_{0}(\Lambda)=\tau<+\infty$. Let $\delta \in(0 ; 1)$ and a natural number $p$ satisfies the condition $\delta /((1-\delta) p)<1, p>1$. We choose an increasing sequence $0<t_{k} \rightarrow \infty$ such that

$$
\bar{L}(\Lambda, \delta)=\lim _{k \rightarrow+\infty} \frac{\lambda\left(t_{k}\right)-\lambda\left(t_{k}(1-\delta)\right)}{-\ln (1-\delta)}
$$

Assume (12). We have

$$
\lambda\left(t_{k}\right)-\lambda\left(t_{k}(1-\delta)\right)=\sum_{l=1}^{p}\left(\lambda\left(t_{k, l}\right)-\lambda\left(t_{k, l}\left(1-\delta_{l}\right)\right)\right), \quad k \in \mathbb{N} .
$$

By Lemma 1 the inequality

$$
\bar{n}_{0}\left(\Lambda, \delta_{l}\right) \leqslant \bar{n}_{0}(\Lambda)=\tau, \quad l=\overline{1, p}
$$

holds true. We fix $\varepsilon>0$. Then according the definition of $\bar{n}_{0}\left(\Lambda, \delta_{l}\right)$ there exists an index $k(\varepsilon)$ such that

$$
\frac{n\left(t_{k, l}, \Lambda\right)-n\left(t_{k, l}\left(1-\delta_{l}\right), \Lambda\right)}{\delta_{l} t_{k, l}} \leqslant \tau+\varepsilon, \quad l=\overline{1, p}, \quad k \geqslant k(\varepsilon) .
$$

Let $k \geqslant k(\varepsilon)$ and $l=\overline{1, p}$. If $t_{k, l}\left(1-\delta_{l}\right)<\lambda_{n} \leqslant t_{k, l}$, then

$$
\frac{1}{\lambda_{n}} \leqslant \frac{1}{t_{k, l}\left(1-\delta_{l}\right)-m \beta}, \quad m=\overline{1, s_{k}},
$$

where $\beta=1 /(\tau+\varepsilon)$ and $s_{k}=\left[(\tau+\varepsilon) t_{k, l} \delta_{l}\right]=\left[(\tau+\varepsilon) \delta t_{k} / p\right]$. Therefore, due to (16) we get

$$
\begin{aligned}
\lambda\left(t_{k, l}\right)-\lambda\left(t_{k, l}\left(1-\delta_{l}\right)\right) & =\sum_{t_{k, l}\left(1-\delta_{l}\right)<\lambda_{n} \leqslant t_{k, l}} \frac{m_{n}}{\lambda_{n}} \leqslant \sum_{m=1}^{s_{k}} \frac{1}{t_{k, l}\left(1-\delta_{l}\right)-m \beta} \\
& =\sum_{m=1}^{s_{k}} \frac{\tau+\varepsilon}{t_{k, l}\left(1-\delta_{l}\right)(\tau+\varepsilon)-m} \leqslant \sum_{m=1}^{s_{k}} \frac{\tau+\varepsilon}{s_{k, l}-m}=\sum_{s=s_{k, l}-s_{k}}^{s_{k, l}-1} \frac{\tau+\varepsilon}{s},
\end{aligned}
$$

where $s_{k, l}=\left[t_{k, l}\left(1-\delta_{l}\right)(\tau+\varepsilon)\right]$. We observe that by (12) with $l=\overline{1, p-1}$

$$
\begin{aligned}
s_{k, l}-s_{k} & >t_{k, l}\left(1-\delta_{l}\right)(\tau+\varepsilon)-(\tau+\varepsilon) t_{k, l} \delta_{l}-1=(\tau+\varepsilon)\left(t_{k, l}-2 t_{k, l} \delta_{l}\right)-1 \\
& =(\tau+\varepsilon)\left(\left(1-\frac{(l-1) \delta}{p}\right) t_{k}-2 \frac{\delta t_{k}}{p}\right)-1 \\
& =(\tau+\varepsilon)\left(\left(1-\frac{((l+1)-1) \delta}{p}\right) t_{k}-\frac{\delta t_{k}}{p}\right)-1=(\tau+\varepsilon)\left(\alpha_{l+1} t_{k}-\frac{\alpha_{l+1} \delta t_{k}}{\alpha_{l+1} p}\right)-1 \\
& =(\tau+\varepsilon)\left(t_{k, l+1}-t_{k, l+1} \delta_{l+1}\right)-1=t_{k, l+1}\left(1-\delta_{l+1}\right)(\tau+\varepsilon)-1 \geqslant s_{k, l+1}-1 .
\end{aligned}
$$

We also note that by the choice of the number $p$ the inequality $s_{k, p}-s_{k} \geqslant 0$ holds true. At that, $s_{k, p}-s_{k}>0$ for all sufficiently large indices $k$.

Thus, in view of (15) and (17), we obtain

$$
\lambda\left(t_{k}\right)-\lambda\left(t_{k}(1-\delta)\right) \leqslant \sum_{l=1}^{p} \sum_{s=s_{k, l}-s_{k}}^{s_{k, l}-1} \frac{\tau+\varepsilon}{s} \leqslant \sum_{s=s_{k, p}-s_{k}}^{s_{k, 1}-1} \frac{\tau+\varepsilon}{s}, \quad k \geqslant k(\varepsilon) .
$$

Since for each natural $l \geqslant 1$ the identity (the Euler formula)

$$
\sum_{s=1}^{l} \frac{1}{s}=\ln l+\gamma+\alpha(l)
$$


holds, where $\gamma$ is the Euler constant and $\alpha(l) \rightarrow 0$ as $l \rightarrow \infty$, by the previous relation we get

$$
\lambda\left(t_{k}\right)-\lambda\left(t_{k}(1-\delta)\right) \leqslant(\tau+\varepsilon) \ln \frac{s_{k, 1}-1}{s_{k, p}-s_{k}}+\tilde{\alpha}(k), \quad k \geqslant k(\varepsilon),
$$

where $\tilde{\alpha}(k) \rightarrow 0, k \rightarrow \infty$. Let us find an upper bound for the expression under the logarithm:

$$
\begin{aligned}
\frac{s_{k, 1}-1}{s_{k, p}-s_{k}} & \leqslant \frac{t_{k, 1}\left(1-\delta_{1}\right)(\tau+\varepsilon)-1}{t_{k, p}\left(1-\delta_{p}\right)(\tau+\varepsilon)-(\tau+\varepsilon) t_{k, p} \delta_{p}-2} \leqslant \frac{t_{k}\left(1-\frac{\delta}{p}\right)(\tau+\varepsilon)}{t_{k, p}\left(1-2 \delta_{p}\right)(\tau+\varepsilon)-2} \\
& \leqslant \frac{t_{k}(\tau+\varepsilon)}{t_{k, p}(\tau+\varepsilon)-2\left(1+t_{k} \frac{\delta}{p}\right)}=\frac{t_{k}(\tau+\varepsilon)}{(1-\delta) t_{k}(\tau+\varepsilon)+t_{k} \frac{\delta}{p}-2\left(1+t_{k} \frac{\delta}{p}\right)} \\
& \leqslant \frac{t_{k}(\tau+\varepsilon)}{(1-\delta) t_{k}(\tau+\varepsilon)-2\left(1+t_{k} \frac{\delta}{p}\right)}=\frac{1}{1-\delta}\left(1-\frac{2\left(1+t_{k} \frac{\delta}{p}\right)}{(1-\delta) t_{k}(\tau+\varepsilon)}\right)^{-1} \\
& =\frac{1}{1-\delta}\left(1-c_{k}(p)\right)^{-1} .
\end{aligned}
$$

By (14) and (18) this implies

$$
\bar{L}(\Lambda, \delta) \leqslant(\tau+\varepsilon) \lim _{k \rightarrow \infty} \frac{\ln (1-\delta)+\ln \left(1-c_{k}(p)\right)}{\ln (1-\delta)}=\tau+\varepsilon+\frac{\ln (1-c(p))}{\ln (1-\delta)},
$$

where

$$
c(p)=\lim _{k \rightarrow \infty} c_{k}(p)=\frac{\delta / p}{(1-\delta)(\tau+\varepsilon)} \rightarrow 0, \quad p \rightarrow \infty
$$

Since the natural number $p$ can be chosen arbitrarily large and $\varepsilon>0$ arbitrarily small, by the above relations we get: $\bar{L}(\Lambda, \delta) \leqslant \tau$. The proof is complete.

Theorem 1 and Lemma 4 imply immediately the following statement.

Theorem 2. The identity

$$
\bar{n}_{0}(\Lambda)=\sup _{\delta \in(0 ; 1)} \bar{L}(\Lambda, \delta)
$$

holds true.

Let us find out the conditions ensuring the identity $\bar{n}_{0}(\Lambda)=\bar{L}(\Lambda)$. Let $\delta \in(0 ; 1)$ and $\bar{n}_{0}(\Lambda, \delta)=\tau<+\infty$. According to the definition of $\bar{n}_{0}(\Lambda, \delta)$, there exists an increasing sequence $0<t_{k} \rightarrow \infty, k \rightarrow \infty$ such that

$$
\tau=\bar{n}_{0}(\Lambda, \delta)=\lim _{k \rightarrow \infty} \frac{n\left(t_{k}\right)-n\left(t_{k}(1-\delta)\right)}{\delta t_{k}} .
$$

It turns out that under condition (20) the points of the sequence $\Lambda$ possess additional uniform distribution in the semi-intervals $\left(t_{k}(1-\delta), t_{k}\right]$ provided we additionally have $\bar{n}_{0}(\Lambda, \alpha)=\tau$, $0<\alpha<\delta$. More precisely the meaning of such uniformity is clarified in the following statement.

Lemma 5. Let $\tau \geqslant 0, \delta \in(0 ; 1)$ and a natural number $p$ satisfy the condition $\delta /((1-\delta) p)<$ 1. Assume that $\bar{n}_{0}(\Lambda, \alpha)=\tau, \alpha \in(0 ; \delta]$, and (20) and (12) hold. Then for each $l=\overline{1, p}$ the limit

$$
\lim _{k \rightarrow \infty} \frac{n\left(t_{k, l}\right)-n\left(t_{k, l}\left(1-\delta_{l}\right)\right)}{\delta_{l} t_{k, l}}
$$

exists and is equal to $\tau$. 
Proof. By the assumption $\bar{n}_{0}(\Lambda, \alpha)=\tau, \alpha \in(0 ; \delta]$. Since $\delta_{l} \leqslant \delta$, by the definition of $\bar{n}_{0}\left(\Lambda, \delta_{l}\right)$ we obtain

$$
\lim _{k \rightarrow \infty} \frac{n\left(t_{k, l}\right)-n\left(t_{k, l}\left(1-\delta_{l}\right)\right)}{\delta_{l} t_{k, l}} \leqslant \bar{n}_{0}\left(\Lambda, \delta_{l}\right) \leqslant \tau, \quad l=\overline{1, p} .
$$

Assume that there exists an index $l_{0}$ obeying

$$
\varliminf_{k \rightarrow \infty} \frac{n\left(t_{k, l_{0}}\right)-n\left(t_{k, l_{0}}\left(1-\delta_{l_{0}}\right)\right)}{t_{k, l_{0}} \delta_{l_{0}}}<\tau .
$$

Let $k_{i}, i \in \mathbb{N}$, be a sequence of natural numbers realizing the bottom limit in (23). Then by (20) we have

$$
\begin{aligned}
\tau & =\lim _{i \rightarrow \infty} \frac{n\left(t_{k_{i}}\right)-n\left(t_{k_{i}}(1-\delta)\right)}{t_{k_{i}} \delta} \\
& =\lim _{i \rightarrow \infty} \frac{1}{t_{k_{i}} \delta}\left(n\left(t_{k_{i}, l_{0}}\right)-n\left(t_{k_{i}, l_{0}}\left(1-\delta_{l_{0}}\right)\right)+\sum_{l=1, l \neq l_{0}}^{p}\left(n\left(t_{k_{i}, l}\right)-n\left(t_{k_{i}, l}\left(1-\delta_{l}\right)\right)\right)\right) .
\end{aligned}
$$

By the choice of subsequence $k_{i}$, the limit

$$
\lim _{i \rightarrow \infty}\left(\left(n\left(t_{k_{i}, l_{0}}\right)-n\left(t_{k_{i}, l_{0}}\left(1-\delta_{l_{0}}\right)\right)\right) / \delta t_{k_{i}}\right)
$$

exists. Therefore, there exists also the limit as $i \rightarrow \infty$ for the expression

$$
\frac{1}{t_{k_{i}} \delta} \sum_{l=1, l \neq l_{0}}^{p}\left(n\left(t_{k_{i}, l}\right)-n\left(t_{k_{i}, l}\left(1-\delta_{l}\right)\right)\right) .
$$

Hence, in view of (12), (22) and (23) we get

$$
\begin{aligned}
\tau & =\lim _{i \rightarrow \infty} \frac{n\left(t_{k_{i}, l_{0}}\right)-n\left(t_{k_{i}, l_{0}}\left(1-\delta_{l_{0}}\right)\right)}{t_{k_{i}} \delta}+\lim _{i \rightarrow \infty} \sum_{l=1, l \neq l_{0}}^{p} \frac{n\left(t_{k_{i}, l}\right)-n\left(t_{k_{i}, l}\left(1-\delta_{l}\right)\right)}{t_{k_{i}} \delta} \\
& =\lim _{i \rightarrow \infty} \frac{n\left(t_{k_{i}, l_{0}}\right)-n\left(t_{k_{i}, l_{0}}\left(1-\delta_{l_{0}}\right)\right)}{t_{k_{i}, l_{0}} \delta_{l_{0}}} \frac{t_{k_{i}, l_{0}} \delta_{l_{0}}}{t_{k_{i}} \delta}+\lim _{i \rightarrow \infty} \sum_{l=1, l \neq l_{0}}^{p} \frac{n\left(t_{k_{i}, l}\right)-n\left(t_{k_{i}, l}\left(1-\delta_{l}\right)\right)}{t_{k_{i}, l} \delta_{l}} \frac{t_{k_{i}, l} \delta_{l}}{t_{k_{i}} \delta} \\
& =\frac{1}{p}\left(\lim _{i \rightarrow \infty} \frac{n\left(t_{k_{i}, l_{0}}\right)-n\left(t_{k_{i}, l_{0}}\left(1-\delta_{l_{0}}\right)\right)}{t_{k_{i}, l_{0}} \delta_{l_{0}}}+\lim _{i \rightarrow \infty} \sum_{l=1, l \neq l_{0}}^{p} \frac{n\left(t_{k_{i}, l}\right)-n\left(t_{k_{i}, l}\left(1-\delta_{l}\right)\right)}{t_{k_{i}, l} \delta_{l}}\right) \\
& <\frac{\tau}{p}+\frac{(p-1) \tau}{p}=\tau .
\end{aligned}
$$

We have obtained the contradiction. Hence, (23) is wrong, that is,

$$
\varliminf_{k \rightarrow \infty} \frac{n\left(t_{k, l_{0}}\right)-n\left(t_{k, l_{0}}\left(1-\delta_{l_{0}}\right)\right)}{t_{k, l_{0}} \delta_{l_{0}}} \geqslant \tau \text {. }
$$

In view of (22) this completes the proof.

Let us prove one more auxiliary statement.

Lemma 6. Let $\bar{n}(\Lambda)=\bar{n}_{0}(\Lambda)=\tau<+\infty$. Then for each $\delta \in(0 ; 1)$ the inequality $\bar{L}(\Lambda, \delta) \geqslant \tau$ holds true.

Proof. Theorem 1 and Lemma 1 imply the identity $\bar{n}_{0}(\Lambda, \alpha)=\tau$ if $\alpha \in(0 ; 1)$. Let $\delta \in(0 ; 1)$, $\varepsilon>0$, a natural number $p$ satisfy the condition $\delta /((1-\delta) p)<1$ and (12) holds. According the definition of $\bar{n}_{0}(\Lambda, \delta)$, there exists a sequence $t_{k}, k \in \mathbb{N}$, such that $(20)$ is true. Then by Lemma 5 , for each $l=\overline{1, p}$, limit (21) exists and is equal to $\tau$. Therefore, there exists an index $k(\varepsilon)$ such that

$$
n\left(t_{k, l}\right)-n\left(t_{k, l}\left(1-\delta_{l}\right)\right)>(\tau-\varepsilon) \delta_{l} t_{k, l}, \quad l=\overline{1, p}, \quad k \geqslant k(\varepsilon) .
$$


Let $k \geqslant k(\varepsilon)$ and $l=\overline{1, p}$. If $t_{k, l}\left(1-\delta_{l}\right)<\lambda_{n} \leqslant t_{k, l}$, then

$$
\frac{1}{\lambda_{n}} \geqslant \frac{1}{t_{k, l}+m \beta}, \quad m=\overline{1, s_{k}}
$$

where

$$
\beta=\frac{1}{\tau-\varepsilon}, \quad s_{k}=\left[(\tau-\varepsilon) t_{k, l} \delta_{l}\right]=\left[\frac{(\tau-\varepsilon) \delta t_{k}}{p}\right] .
$$

Therefore, in view of (24) we obtain

$$
\begin{aligned}
\lambda\left(t_{k, l}\right)-\lambda\left(t_{k, l}\left(1-\delta_{l}\right)\right) & =\sum_{t_{k, l}\left(1-\delta_{l}\right)<\lambda_{n} \leqslant t_{k, l}} \frac{m_{n}}{\lambda_{n}} \geqslant \sum_{m=1}^{s_{k}} \frac{1}{t_{k, l}+m \beta} \\
& =\sum_{m=1}^{s_{k}} \frac{\tau-\varepsilon}{t_{k, l}(\tau-\varepsilon)+m} \geqslant \sum_{m=1}^{s_{k}} \frac{\tau-\varepsilon}{s_{k, l}+1+m}=\sum_{s=s_{k, l}+2}^{s_{k, l}+s_{k}+1} \frac{\tau-\varepsilon}{s},
\end{aligned}
$$

where $s_{k, l}=\left[t_{k, l}(\tau-\varepsilon)\right]$. We note that by (12)

$$
\begin{aligned}
s_{k, l} & \leqslant t_{k, l}(\tau-\varepsilon)=(\tau-\varepsilon)\left(1-\frac{(l-1) \delta}{p}\right) t_{k} \\
& =(\tau-\varepsilon)\left(\left(1-\frac{((l+1)-1) \delta}{p}\right) t_{k}+\frac{\delta t_{k}}{p}\right)=(\tau-\varepsilon)\left(\alpha_{l+1} t_{k}+\frac{\delta t_{k}}{p}\right)-1 \\
& =(\tau-\varepsilon)\left(t_{k, l+1}+t_{k, l+1} \delta_{l+1}\right) \leqslant s_{k, l+1}+s_{k}+2 .
\end{aligned}
$$

Thus, in view of (15) and (25) we have

$$
\begin{aligned}
\lambda\left(t_{k}\right)-\lambda\left(t_{k}(1-\delta)\right) & \geqslant \sum_{l=1}^{p} \sum_{s=s_{k, l}+2}^{s_{k, l}+s_{k}+1} \frac{\tau-\varepsilon}{s} \geqslant \sum_{s=s_{k, p}+2}^{s_{k, 1}+s_{k}+1} \frac{\tau-\varepsilon}{s}-\sum_{l=1}^{p}\left(\frac{1}{s_{k, l}}+\frac{1}{s_{k, 1}+1}\right) \\
& \geqslant \sum_{s=s_{k, p}+2}^{s_{k, 1}+s_{k}+1} \frac{2 p}{s}-\frac{2 p}{t_{k}(1-\delta)(\tau-\varepsilon)}, \quad k \geqslant k(\varepsilon) .
\end{aligned}
$$

By Euler formula this implies:

$$
\lambda\left(t_{k}\right)-\lambda\left(t_{k}(1-\delta)\right) \geqslant(\tau-\varepsilon) \ln \frac{s_{k, 1}+s_{k}+1}{s_{k, p}+2}--\frac{2 p}{t_{k}(1-\delta)(\tau-\varepsilon)}+\tilde{\alpha}(k), \quad k \geqslant k(\varepsilon),
$$

where $\tilde{\alpha}(k) \rightarrow 0, k \rightarrow \infty$. Let us estimate from below the expression under the logarithm:

$$
\begin{aligned}
\frac{s_{k, 1}+s_{k}+1}{s_{k, 1}+2} & \geqslant \frac{t_{k, 1}(\tau-\varepsilon)+(\tau-\varepsilon) \delta \frac{t_{k}}{p}-1}{t_{k, p}(\tau-\varepsilon)+2} \\
& \geqslant \frac{t_{k}(\tau-\varepsilon)-1}{t_{k}(1-\delta)(\tau-\varepsilon)+(\tau-\varepsilon) \delta \frac{t_{k}}{p}+2}=c_{k}(p) .
\end{aligned}
$$

In view of the definition of $\bar{L}(\Lambda, \delta)$ and (26) this yields

$$
\bar{L}(\Lambda, \delta) \geqslant(\tau-\varepsilon) \lim _{k \rightarrow \infty} \frac{c_{k}(p)}{-\ln (1-\delta)}=(\tau-\varepsilon) \frac{\ln c(p)}{-\ln (1-\delta)},
$$

where

$$
\begin{aligned}
c(p) & =\lim _{k \rightarrow \infty} c_{k}(p)=\lim _{k \rightarrow \infty} \frac{t_{k}(\tau-\varepsilon)-1}{t_{k}(1-\delta)(\tau-\varepsilon)+(\tau-\varepsilon) \delta \frac{t_{k}}{p}+2} \\
& =\frac{1}{(1-\delta)+\frac{\delta}{p}} \rightarrow \frac{1}{1-\delta}, \quad p \rightarrow \infty .
\end{aligned}
$$


Since the natural number $p$ can be chosen arbitrarily large and $\varepsilon>0$ can be arbitrarily small, we get: $\bar{L}(\Lambda, \delta) \geqslant \tau$. The proof is complete.

Theorem 3. Let $\bar{n}_{0}(\Lambda)=\tau<+\infty$. Then the following statements are equivalent:

1) $\bar{n}_{0}(\Lambda, \delta)=\tau, \delta \in(0 ; 1)$;

2) $\bar{n}(\Lambda)=\tau$;

3) $\bar{L}(\Lambda, \delta)=\tau, \delta \in(0 ; 1)$;

4) $\bar{L}(\Lambda, \delta)=\tau$.

Proof. The implication 1) $\Longrightarrow 2$ ) follows Lemma 2 .

$2) \Longrightarrow 3$ ). Let $\bar{n}(\Lambda)=\tau$. Then by the assumption and Lemma 6 the inequality $\bar{L}(\Lambda, \delta) \geqslant \tau$, $\delta \in(0 ; 1)$, holds true. Moreover, the assumption and Lemma 4 implies also the inequality $\bar{L}(\Lambda, \delta) \leqslant \tau, \delta \in(0 ; 1)$. Thus, $\bar{L}(\Lambda, \delta)=\tau, \delta \in(0 ; 1)$.

The implication 3$) \Longrightarrow 4$ ) is due to formulae (2) and (3).

Finally, by Lemma 1 and in view of the assumption we get the implication 4$) \Longrightarrow 1$ ). The proof is complete.

Remark. It follows from Theorem 3 that under at least one of the relations $\bar{n}_{0}(\Lambda)=\bar{n}(\Lambda)<$ $+\infty$ and $\bar{n}_{0}(\Lambda)=\bar{L}(\Lambda)<+\infty$ the identities $\bar{n}_{0}(\Lambda)=\bar{n}(\Lambda)=\bar{L}(\Lambda)$ hold. A natural question arises: whether these identities are due to the relation $\bar{n}(\Lambda)=\bar{L}(\Lambda)<+\infty$ ? The answer is negative. Let us consider the corresponding example

Let $0<R_{k} \rightarrow \infty$ and $R_{k+1} / R_{k} \rightarrow \infty, k \rightarrow \infty$. We define $\Lambda=\bigcup_{k \in \mathbb{N}}\left(\Lambda_{k} \cup \tilde{\Lambda}_{k}\right)$, where $\Lambda_{k}$ is the set of all natural numbers in the interval $\left(R_{2 k} ; R_{2 k+1}\right)$ and the multiplicity of each number is equal to and $\tilde{\Lambda}_{k}=\left\{R_{2 k+2}, p_{k}\right\}$, where $p_{k}=\left[R_{2 k+2}\right]-\left[R_{2 k+1}\right], k \in \mathbb{N}$, is the multiplicity of the number $R_{2 k+2}$. By the choice of the numbers $R_{k}$ we have

$$
\lim _{k \rightarrow \infty} \frac{n\left(R_{2 k+1}, \Lambda\right)}{R_{2 k+1}} \geqslant \lim _{k \rightarrow \infty} \frac{R_{2 k+1}-R_{2 k}}{R_{2 k+1}}=1
$$

We note that by the construction $n(t, \Lambda) \leqslant t, t>0$. This is why

$$
\bar{n}(\Lambda)=\varlimsup_{t \rightarrow+\infty} \frac{n(t, \Lambda)}{t} \leqslant \lim _{t \rightarrow+\infty} \frac{t}{t}=1 .
$$

Therefore, $\bar{n}(\Lambda)=1$. Let $\delta \in(0 ; 1)$. By the Euler formula we obtain:

$$
\begin{aligned}
\bar{L}(\Lambda, \delta) & \geqslant \lim _{k \rightarrow \infty} \frac{\lambda\left(R_{2 k+1}\right)-\lambda\left(R_{2 k+1}(1-\delta)\right)}{-\ln (1-\delta)} \\
& =\lim _{k \rightarrow \infty} \frac{\ln \left[R_{2 k+1}\right]-\ln \left[R_{2 k+1}(1-\delta)+1\right]}{-\ln (1-\delta)}=\lim _{k \rightarrow \infty} \frac{-\ln (1-\delta)}{-\ln (1-\delta)}=1 .
\end{aligned}
$$

By Lemma 1 and formula (2) this implies the identity $\bar{L}(\Lambda)=\bar{n}(\Lambda)$. At the same time we have

Thus,

$$
\bar{n}_{0}(\Lambda, \delta)=\lim _{k \rightarrow \infty} \frac{n\left(R_{2 k+2}, \Lambda\right)-n\left(R_{2 k+2}(1-\delta), \Lambda\right)}{\delta R_{2 k+2}}=\lim _{k \rightarrow \infty} \frac{\left[R_{2 k+2}\right]-\left[R_{2 k+1}\right]}{\delta R_{2 k+2}}=\frac{1}{\delta} .
$$

$$
\bar{n}_{0}(\Lambda)=\lim _{\delta \rightarrow 0+} \bar{n}_{0}(\Lambda, \delta) \lim _{\delta \rightarrow 0+} \frac{1}{\delta}=+\infty
$$

We are going to apply the obtained result to the issue on the completeness of the system of exponential monomials with positive exponents in convex domains in the complex plane.

We recall that an entire function $f$ is called an entire function of exponential type if for some positive constants $A, B$ depending on $f$ and for each $z \in \mathbb{C}$ the inequality $\ln |f(z)| \leqslant A|z|+B$ holds. It indicator (upper indicator) is the function

$$
h_{f}(\varphi)=\varlimsup_{r \rightarrow+\infty} \frac{\ln \left|f\left(r e^{i \varphi}\right)\right|}{r}, \quad \varphi \in(-\pi ; \pi] .
$$


By Pólya theorem, see, for instance, [10, Ch. I, Sect. 5, Thm. 5.4], the indicator coincides with the support function $H_{K}(\varphi)$ of some convex compact set $K \subset \mathbb{C}$ called the indicator diagram of the function $f[10$, Ch. I, Sect. 5]:

$$
h_{f}(\varphi)=H_{K}(\varphi)=\sup _{z \in K} \operatorname{Re}\left(z e^{-i \varphi}\right) .
$$

The compact set complex conjugate for the compact set $K$ is called the conjugate diagram of the function $f$.

Let $D$ be a convex domain. By the Khan-Banach theorem, the system $\mathcal{E}(\Lambda)=\left\{z^{k} e^{\lambda_{n} z}\right\}_{n=1, k=1}^{\infty, m_{n}}$ is incomplete in the space $H(D)$ if and only if there exists a non-zero linear continuous functional $\nu \in H^{*}(D)$ vanishing on all elements of this system.

Let $P_{D}$ be the space of all entire functions of exponential type, whose conjugate diagrams are located in $D$. It is equipped with the standard topology of inductive limit, see, for instance, [9, Ch. III, Sect. 12, Subsect. 7]. The Laplace transform $f(\lambda)=\nu(\exp (\lambda z)), \nu \in H^{*}(D)$, makes an isomorphism of the linear topological spaces $H^{*}(D)$ and $P_{D}$, see, for instance, [9, Ch. III, Sect. 12, Thms. 12.3, 12.13].

Thus, the incompleteness of $\mathcal{E}(\Lambda)$ in the domain $D$ is equivalent to the existence of a function $0 \neq f \in P_{D}$ vanishing at the points $\lambda_{n}$ with the multiplicities at least $m_{n}, n \in \mathbb{N}$.

We shall say that a convex domain $D$ is vertically balanced if

$$
H_{D}\left(-\frac{\pi}{2}\right)+H_{D}\left(\frac{\pi}{2}\right)=d(D)
$$

where $H_{D}$ is the support function of the domain $D$ and $d(D)$ is its vertical diameter. It is easy to show that vertically balanced domains are, for instance, those having a vertical or a horizontal symmetry axis.

The next statement generalizes the result by A.F. Leontiev and B.Ya. Levin mentioned in the beginning of the work; the generalization is made for verically balanced convex domains.

Theorem 4. Let $\bar{n}_{0}(\Lambda)=\tau<+\infty$. Then the following statements are equivalent:

1) $\bar{n}(\Lambda)=\tau$;

2) the system $\mathcal{E}(\Lambda)$ is incomplete in each convex domain $D$ with the vertical diameter $d(D)>$ $2 \pi \tau$ and is complete in each vertically balanced convex domain $D$ with the vertical diameter $d(D) \leqslant 2 \pi \tau$.

Proof. Assume that the sequence $\Lambda$ has a finite maximal density $\tau$. Let us prove the implication $1 \Longrightarrow 2$. Since $\bar{n}_{0}(\Lambda)=\tau$, by Lemma 2.1 in work [11] (see also [12, Lm. 5]) there exists a measurable sequence $\Lambda^{\prime}=\left\{\mu_{k}, p_{k}\right\}_{k=1}^{\infty}$ with the density $\tau$ such that the relations

$$
\mu_{k_{n}}=\lambda_{n}, \quad m_{n} \leqslant p_{k_{n}}, \quad n \in \mathbb{N},
$$

hold true, where $\left\{k_{n}\right\}_{n=1}^{\infty}$ is a some subsequence of natural numbers. Since the arguments of the terms in the sequence do not influence its density, we can assume that $\mu_{k}>0, k \in \mathbb{N}$. We consider the function

$$
f(\lambda)=\prod_{k=1}^{\infty}\left(1-\frac{\lambda^{2}}{\mu_{k}^{2}}\right)^{p_{k}} .
$$

It vanishes at the points $\mu_{k}$ with the multiplicities $p_{k}, k \geqslant 1$ and in particular, at the points $\lambda_{n}$ with the multiplicities at least $m_{n}, n \geqslant 1$. Since $\Lambda^{\prime}$ has the density $\tau$, then $f(\lambda)$ is an entire function of exponential type with the indicator $h_{f}(\varphi)=\pi \tau|\sin \varphi|$ [10, Ch. I, Sect. 5, Thm. 5.9], [5, Ch. II, Sect.1, Thm. 2]. It coincides with the support function $H_{K}(\varphi)$ of the segment of the imaginary axis $[-i \pi \tau ; i \pi \tau]$. Therefore, this segment is the indicator diagram of the function $f$ and hence, also its conjugate diagram.

Let $D$ be a convex domain with a vertical diameter $d(D)>2 \pi \tau$. Then in the domain $D$ there exist points $z_{1}=x_{0}+i y_{1}$ and $z_{2}=x_{0}+i y_{2}$ such that

$$
\left|z_{2}-z_{1}\right|=\left|y_{2}-y_{1}\right|=2 \pi \tau \text {. }
$$


Thus, the domain $D$ contains the vertical segment $\left[z_{1}, z_{2}\right]$ of the length $2 \pi \tau$. We let $\tilde{f}(\lambda)=$ $f(\lambda) e^{z_{0} \lambda}$, where $z_{0}=\left(z_{2}-z_{1}\right) / 2$. It is to observe that the indicator diagram of this function coincides with the segment $\left[\bar{z}_{1} ; \bar{z}_{2}\right]$ complex conjugate with the segment $\left[z_{1} ; z_{2}\right]$. This is why the conjugate diagram of this function coincides with the segment $\left[z_{1}, z_{2}\right]$, that is, $\tilde{f} \in P_{D}$. Therefore, in view of the said above, the system $\mathcal{E}(\Lambda)$ is incomplete in the domain $D$. This proves the first part of Statement 2).

Let us prove the second part. Let $D$ be a vertically balanced domain with a vertical diameter $d(D) \leqslant 2 \pi \tau$. Since $\bar{n}(\Lambda)=\bar{n}_{0}(\Lambda)=\tau$, by Theorem 3 the identity $\bar{L}(\Lambda)=\tau$ holds.

Assume that the system $\mathcal{E}(\Lambda)$ is incomplete in the domain $D$. Then there exists a function $0 \neq f \in P_{D}$ vanishing at the points $\lambda_{n}$ with the multiplicity at least $m_{n}, n \geqslant 1$. Its conjugate diagram $K$ is located in the domain $D$, that is, the inequality $H_{K}(\varphi)<H_{D}(\varphi), \varphi \in(-\pi ; \pi]$, holds true. This is why in view of the vertical balance of $D$ we obtain

$$
H_{K}\left(-\frac{\pi}{2}\right)+H_{K}\left(\frac{\pi}{2}\right)<H_{D}\left(-\frac{\pi}{2}\right)+H_{D}\left(\frac{\pi}{2}\right)=d(D) \leqslant 2 \pi \tau .
$$

We let

$$
y_{0}=-\frac{1}{2}\left(H_{K}\left(\frac{\pi}{2}\right)-H_{K}\left(-\frac{\pi}{2}\right)\right), \quad \tilde{f}(z)=f(z) e^{i y_{0} z} .
$$

Let $\tilde{K}$ be the conjugate diagram of the function $\tilde{f}$. By the choice of the number $y_{0}$ we have

$$
H_{\tilde{K}}\left(-\frac{\pi}{2}\right)=H_{\tilde{K}}\left(\frac{\pi}{2}\right) \text {. }
$$

Therefore, in view of (28) we obtain

$$
H_{\tilde{K}}\left( \pm \frac{\pi}{2}\right)<\pi \tau
$$

The same inequality is obviously true for the indicator diagram of the function $\tilde{f}$. This is why by $(27)$

$$
h_{\tilde{f}}\left(\mp \frac{\pi}{2}\right)<\pi \tau
$$

Then by Theorem 6.2 in [1] the inequality $\bar{L}(\Lambda)<\tau$ holds true. We have obtained the contradiction. Thus, system $\mathcal{E}(\Lambda)$ is complete in the domain $D$.

Now we are going to prove the implication 2$) \Longrightarrow 1$ ). By Theorem 3 it is sufficient to show that $\bar{L}(\Lambda)=\tau$. Assume that $\bar{L}(\Lambda)<\tau$. Then by Theorem 6.2 of work [1], there exists an entire function $f \neq 0$ of exponential type vanishing at the points $\lambda_{n}$ with the multiplicities at least $m_{n}, n \geqslant 1$, such that

$$
h_{f}\left(\mp \frac{\pi}{2}\right)<\pi \tau \text {. }
$$

Therefore, the indicator diagram of the function $f$ (and its conjugate diagram) lies in the strip $D=\{z:|\operatorname{Im} z|<\pi \tau\}$. This means that the system $\mathcal{E}(\Lambda)$ is incomplete in this strip. We have obtained the contradiction with Statement 2 since the convex domain $D$ is vertically balanced and has the vertical diameter $d(D)=2 \pi \tau$. Thus, our assumption is wrong, that is, the inequality $\bar{L}(\Lambda) \geqslant \tau$ holds true. It remains to observe that by Lemma 1 we have $\bar{L}(\Lambda) \leqslant \bar{n}_{0}(\Lambda)=\tau$. The proof is complete.

In conclusion we note that according to Remark 1 to Lemma 1 , a measurable sequence $\Lambda$ always satisfies the identity $\bar{n}(\Lambda)=\bar{n}_{0}(\Lambda)$. The opposite is wrong. Let us provide the corresponding example. Let $0<R_{k} \rightarrow \infty, k \rightarrow \infty$, and $R_{k+1} / R_{k} \rightarrow \infty, k \rightarrow \infty$. We define $\Lambda=\bigcup_{k \in \mathbb{N}} \Lambda_{k}$, where $\Lambda_{k}$ is the set of all natural numbers in the segment $\left(R_{2 k} ; R_{2 k+1}\right), k \in \mathbb{N}$, the multiplicty of each number is equal to one. By the choice of the numbers $R_{k}$ we have:

$$
\bar{n}(\Lambda)=\varlimsup_{t \rightarrow+\infty} \frac{n(t, \Lambda)}{t} \geqslant \lim _{k \rightarrow \infty} \frac{n\left(R_{2 k+1}, \Lambda\right)}{R_{2 k+1}} \geqslant \lim _{k \rightarrow \infty} \frac{R_{2 k+1}-R_{2 k}}{R_{2 k+1}}=1 .
$$


Let $\delta \in(0 ; 1)$. Then

$$
\bar{n}_{0}(\Lambda, \delta)=\lim _{k \rightarrow \infty} \frac{n\left(R_{2 k+1}, \Lambda\right)-n\left(R_{2 k+1}(1-\delta), \Lambda\right)}{\delta R_{2 k+1}}=\lim _{k \rightarrow \infty} \frac{\delta R_{2 k+1}}{\delta R_{2 k+1}}=1 .
$$

By Lemma 1 this implies: $\bar{n}_{0}(\Lambda)=\bar{n}(\Lambda)$. At the same time, we have

$$
\underline{n}(\Lambda)=\lim _{k \rightarrow \infty} \frac{n\left(R_{2 k}, \Lambda\right)}{R_{2 k}} \leqslant \lim _{k \rightarrow \infty} \frac{R_{2 k-1}}{R_{2 k}}=0 .
$$

Thus, $\underline{n}(\Lambda)<\bar{n}(\Lambda)$, that is, the sequence $\Lambda$ has no density.

\section{BIBLIOGRAPHY}

1. P. Malliavin, L.A. Rubel. On small entire functions of exponential type with given zeros // Bull. Soc. Math. France. 89, 175-201 (1961).

2. B.N. Khabibullin. On the growth of entire functions of exponential type along the imaginary axis // Matem. Sborn. 180:5, 706-719 (1989). [Math. USSR-Sb. 67:1, 149-163 (1990).]

3. A.I. Abdulnagimov, A.S. Krivosheyev. Properly distributed subsequence on the line // Ufimskij Matem. Zhurn. 7:1, 3-12 (2015). [Ufa Math. J. 7:1, 3-12 (2015).]

4. A.F. Leont'ev. On completeness of a system of exponential functions in a curvilinear strip // Matem. Sborn. 36:3, 555-568 (1955). (in Russian).

5. B.Ya. Levin. Distribution of zeros of entire functions. Gostekhizdat, Moscow. 1956. [Amer. Math. Soc., Providence, RI. 1980.]

6. G. Pólya. Untersuchungen über Lücken und Singularitäten von Potenzreihen // Math. Zeit. 29:1, 549-640 (1929). (in German).

7. P. Koosis. The logarithmic integral I. Cambridge Univ. Press, Cambrige (1997).

8. A.I. Abdulnagimov, A.S. Krivosheyev. Properly distributed subsets in complex plane // Alg. Anal. 28:4, 1-46 (2016). [St.-Petersburg Math. J. 28:4, 433-464 (2017).]

9. V.V. Napalkov. Convolution equations in multi-dimensional spaces. Nauka, Moscow (1982). (in Russian).

10. A.F. Leont'ev. Entire functions. Exponential series. Nauka, Moscow (1983). (in Russian).

11. A.S. Krivosheev, O.A. Krivosheeva. Fundamental principle and a basis in invariant subspaces // Matem. Zamet. 99:5, 684-697 (2016). [Math. Notes. 99:5, 685-696 (2016).]

12. A.S. Krivosheyev, O.A. Krivosheyeva. A closedness of set of Dirichlet series sums // Ufimskij Matem. Zhurn. 5:3, 96-120 (2013). [Ufa Math. J. 5:3, 94-117 (2013).]

Alexandr Sergeevich Krivosheyev,

Institute of Mathematics, Ufa Scientific Center, RAS,

Chernyshevsky str. 112,

450008, Ufa, Russia

E-mail: kriolesya2006@yandex.ru

Arsen Fanilevich Kuzhaev,

Bashkir State University,

Zaki Validi str. 32,

450076, Ufa, Russia

E-mail: arsenkuzh@outlook.com 